Journal of ISMAC (2019)

Vol.01/ No. 03

Pages: 188-205

http://irojournals.com/iroismac/

DOI: https://doi.org/10.36548/jismac.2019.3.006

\title{
VIRTUAL STRUCTURE FOR SUSTAINABLE WIRELESS NETWORKS IN CLOUD SERVICES AND ENTERPRISE INFORMATION SYSTEM
}

\author{
Dr. Jennifer S. Raj, \\ Professor, \\ Department of ECE, \\ Gnanamani College of Technology, \\ Namakkal, India. \\ Email: jennifer.raj@gmail.com
}

\author{
Dr. S. Smys, \\ Professor, \\ Department of ECE, \\ RVS Technical Campus, \\ Coimbatore, India. \\ Email: smys375@gmail.com
}

\begin{abstract}
The wireless network virtualization for the cloud and the enterprise information system would enable maximum utilization of resources, causing an enhancement in the enterprise information system efficiency, by sharing the infrastructure among multiple applications or service provisioning's. The physical wireless structure of the network and the radio resources are split and shared among different applications by abstraction and isolation to provide a cost effective and efficient handling of the enormous amount of the services. Further the virtualization also provides a migration to the latest products and the technologies at ease. In spite of all these potentials there are several challenges that are to be handled before embedding the virtual wireless network over the physical infrastructure. The paper focuses on developing a virtual wireless network that are sustainable(VSWN) for the cloud and enterprise information system involving the software defined networks that includes network function virtualizing and deep packet inspection for improving the agility and the flexibility of the Cloud enterprise information system. The further validation of the proposed model using the network simulator shows throughput enhancements with cost efficiency when compared to the other wireless network management of cloud.
\end{abstract}

Keywords: Virtual wireless networks, enterprise information system, cloud computing, SDN, NFV, DPI

\section{Introduction}

The enterprise information system is the convergence of the different application, protocols and formats in collecting, processing, storing and sharing of information's to enhance the business proceedings by combining the business, people, framework and the technology. The EIS offers a technical platform for the purpose of interchange, interconnection and distributed computing for the co-ordination of the business process for a strong substructure. Remaining as a part of the concern the enterprise information system aims to fulfill the operational and the basic objectives of the organization. The enterprise information system that are incorporated into the infrastructure of the 
Journal of ISMAC (2019)

Vol.01/ No. 03

Pages: 188-205

http://irojournals.com/iroismac/

DOI: https://doi.org/10.36548/jismac.2019.3.006

organization has its effect on the planning, functioning and the execution of the tasks in the organization and are supposed to have a prolonged operating period so as to increase the productivity and the competitions between the organizations. The manifestation of the cloud computing or the cutting edge technologies into the EIS has caused the possible extension of the enterprise information system to show efficiency, proficiency and ambitiousness. More over the cloud paradigm shows a principle change in the creating, developing, deploying, scaling, updating, and maintaining the services of the enterprise information systems. The cloud computing is a promising technology in delivering all the services of the information system of the enterprise at a minimum cost. Cloud paradigm involves certain inclinations in the service rendering of the enterprise information system, where one of it includes the efficient processing of the information, in which the power utilizations of the computing resources are efficiently handled. The other one involves in enhancing the business activity by the use of interactive mobile service application, computational intensive business analytics and parallel processing. These inclinations enable the EIS services handled by cloud to be more convincing even for very small industries by the immediate access to the hardware and accurate information provisioning. Further the use of virtual wireless network that are sustainable would pave way for the new direction in the cloud computing service extension for the enterprise information system.

The virtual wireless network for the cloud and the enterprise information system would enable more and more cost effective transmission of information over the shared infrastructure. They virtualization of the wireless network ranges over a wide scope from allocation of spectrum to virtualization of the infrastructure. The physical virtualized infrastructure that are shared among different number of service provisioning are abstracted and isolated in aid of supporting the sharing among the multiple service provisioning. It could be considered as the procedure to split the network for the efficient handling of the monumental growth in the service rendering and the congestion level. The virtualization causes a separation of the network from the services it does, to provide a maximum resources utilization by allowing multiple applications to run on the same infrastructure.

In spite of the potentials envisioned from the wireless network virtualization there are several challenges incurred in the virtualization of the network. The main challenges to be addressed are resource sharing, resource allocation, the separation of the services and the structure, controlling, motility and connectivity management, further the embedding of the virtual wireless network into the physical infrastructure inclusive of the additional devices that are capable of self-adapting with context awareness are more tedious and chaotic

So the paper proposes a frame of virtual structure for the wireless network that are sustainable for the cloud and the enterprise information system. The proposed method focuses on the virtualization of the network to using the SDN (software defined networks), NFV (network virtualization function), and DPI (deep packet inspection) for improving the agility and the flexibility of the network in the enterprise information system that is based on the cloud computing. 
Journal of ISMAC (2019)

Vol.01/ No. 03

Pages: $188-205$

http://irojournals.com/iroismac/

DOI: https://doi.org/10.36548/jismac.2019.3.006

The remaining of the paper is arranged with 2 related works, 3 proposed work, 4 result and analysis and 5 conclusion.

\section{Related works}

Rajesh et al [1], the control module development by merging the cloud and the wireless sensor networks for realization of applications with improved reliability, adaptability and elasticity in the real-time. Irish et al [2] the data collection methodology with dynamic sink mobility is proposed in this paper to have a full sink mobility utilization and random move with efficient data collection. Khan et al [3] the virtualization of wireless sensor network is presented in this paper for the purpose of availing the same infrastructure to multiple functions of the internet of things. The potential sharing of the infrastructure with basics of the visualization is discussed in this paper gives the clear step on virtualization process for the WSN as a survey Fontes et al [4] the paper proposes an effective wireless sensor network usage that is afforded by virtualization to minimize the deploying of more number of WSN, this is done by introducing a mini-net wireless network involving the software defined network. Khan et al [5] the wireless network sharing among different number of applications can be used for reducing the enormous WSN distribution using the virtualization process by semantic application using the data annotation architecture. Nkomo et al [6] the network virtualization in the industrial Iot is focused in this paper. The virtual networking is proceeded without affecting the logical links in the network, the challenges and the advantages of virtualization of the wireless sensor network in the in the industrial IOT is discussed by the author. Khan et al [7] the evolution computation based on the genetic algorithm is proposed in this paper for the data annotation for the virtualizing of the WSN to be distributed among concurrent multiple applications. Arjun et al [8] the high quality disaster alerts for and the weather reports at an economical rate is provided by the virtualizing of the wireless sensor network using the sensor that are virtual and the concept of the overlay. Raee et al [9] the static version of the integer linear programming is used for the purpose of the wireless virtualization to be deployed among numerous of application in solving the problems under different situations with minimized energy usage. Kaiwartyaet al [10] the internet of things that is used for service provisioning is aided with the wireless sensor network that is fault tolerant using the genetic based algorithm that is adopted nominated and sorted. Han et al [11] provides a brief overview of the network function virtualization that is involved in the flexible functioning of the network Kumar et al [12] the virtualization in the WSN is used in the commercial applications for offering more cost effective solutions with security and manageability. Niebert et al [13] the embedded network virtualization and the architecture is presented to provide an information centric networking for future internet communications. Liang et al [14] the WSN and information centric communication are involved in the network caching and the resource allocation by integrating the WSN with ICN in 5G wireless mobile network. Feng et al [15] the $5 \mathrm{G}$ mobile networks co-ordination and management among heterogeneous network is made possible by the wireless network virtualization to offer flexibility in resource allocation with efficiency that is high. Romero et al [16] the paper provides the future perception on the enterprise information system by providing a short history on the 
Journal of ISMAC (2019)

Vol.01/ No. 03

Pages: 188-205

http://irojournals.com/iroismac/

DOI: https://doi.org/10.36548/jismac.2019.3.006

enterprise information system and the various aspects of the engineering involved in the EIS design. Petrarch et al [17] the concept of enterprise data management and enterprise information system has shifted to new epoch by the IOT and cloud by converging different data sources through the mapping and the rule system to provide a real time and consistent data. Fang et al [18] the integrated information system combined with the cloud computing and the IOT is used in the monitoring of the climatic and the environmental changes. Zhang et al [19] the mass storage problems and the high amount of data to be processed in the enterprise level is overcome using the cloud storage device that could afford a large storage capacity and the flexible extension of the storage. Jun et al [20] the collaboration in the supply chain is made easy using the cloud computing by developing a supply chain management mode and a supply chain transfer mode and also presents the research problems in the supply chain management.

\section{Proposed Work}

The wireless network that are virtualized, affords the resources sharing by abstracting and isolating the hardware and the software, to allow multiple clients to efficiently make use of the same network resources, for operating different protocols without any disagreement. The virtualization offers much benefits by a cost effective service provisioning, acquired by resource sharing and the easy relocation of the clients to latest product and the technologies. Though incorporated with much potency the embedding of the virtual network over the physical frame work becomes quiet tedious as it has to face many challenges such as abstraction, isolation resource allocation along with other part that come with self-adaptability and context awareness to handle all the services without disruptions.

So the proposed method uses the software defined network including network function virtualization and deep packet inspection for framing the virtual structure of the network that are sustainable and embedding it on the physical infrastructure for creating virtual wireless structures that are sustainable for the cloud services and the enterprise information system.

\subsection{Software Defined networking for Wireless Network}

The SDN in wireless network, is the ingenious concept of networking paradigm that separates the system configuration and the management layer from the forwarding layer to provide an enhanced network performance by changing the decentralized network functionality to centralized network functionality, the centralized network functionality offers a centralized management of the frame work and removes the control plane from the hardware layer of the network and does a software implementation for it, to have a flexible administration possible, by the programmable access activation. This dynamic access and the administration as the result of software implemented control plane makes 
Journal of ISMAC (2019)

Vol.01/ No. 03

Pages: 188-205

http://irojournals.com/iroismac/

DOI: https://doi.org/10.36548/jismac.2019.3.006

the infrastructure underlying to be inattentive of the application and the services rendered by the network and thus enabling the administration to adjust the traffic flow, that is wide in the network, to meet the dynamic needs by the consistent network management. The programming capability enabled by the network on the traffic handling and the devices in the network makes the network flexible and efficient than the conventional networking where all the layers are converged into a firmware.

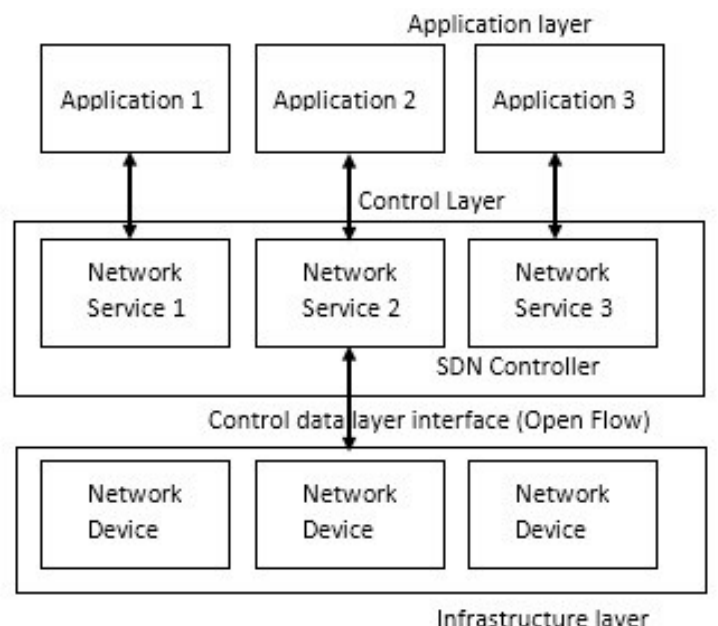

Fig 1. SDN

The Fig1 shows the software implemented control layer that is placed between the application and the network devices, in a centralized manner on deciding the destination of the traffic flow, appearing like a single logic switch, maintaining the global view of the network without the need for the independent accessing and configuring of the separate hardware devices in the network and the understanding variety of network protocols that includes the implementation of the same. The software based control layer takes the vital part in the SDN and acts as the operating system for the SDN allowing an improved automated management of network and easy integration of applications by minimizing the configurations that are manual. This instructs the switches based on the protocol (open flow protocol) for directing the forwarding of the data's to its preferred destination by a higher level routing policies. This type of networking causes considerable saving in the time, resources and cost due to its programmable centralized control of the network behavior.so the software defined network is designed to offers, the enabling and disabling of the effective service rendering on demand, the instigating of the innovative services and business applications, the utilizing of the enhanced performance and reduced -cost by the off the shelf hardware devices of the network such as gateways, switches, proxy servers etc., the replacing of the routers that are complex with the simple devices, and provides more services by aiding diverse applications. 
Journal of ISMAC (2019)

Vol.01/ No. 03

Pages: 188-205

http://irojournals.com/iroismac/

DOI: https://doi.org/10.36548/jismac.2019.3.006

The SDN though possess high potency as mentioned above come across certain challenges in the complete replacing of the existing devices of the network, lack of context awareness, and efficient management evaluating the complete traffic flow. So the SDN entails the NFV and DPI that complements the SDN with the necessary elasticity and scalability for sustainable virtual wireless network.

\subsection{Network Function Virtualization in Aid to SDN.}

The network function virtualization contributes added features to the software defined networking, to emphasize its quality. Independent on the concepts of the software defined networking the network-function virtualization separates the hardware and the software to enable the network deployment that is flexible with dynamic operation capabilities. The network-function virtualization provides a set of resources on computing and networking for the execution of the virtualized instances of services and applications with a faster service provisioning that are cost effective by eluding the procurement of the hardware devices that are dedicated. The network-function virtualization improves the operation and the managing of the networks with fewer operating troubles.

The NFVs exchange the expensive individual or group of hardware appliances with common servers that use software's that are capable of providing a bundle of different virtual functions as shown in Fig 2. The NFVs provide the firewalls and the load balancers that help in the securing of the network and the even workload sharing in the network respectively with more rapidness and at ease. Apart from this the network-function virtualization also

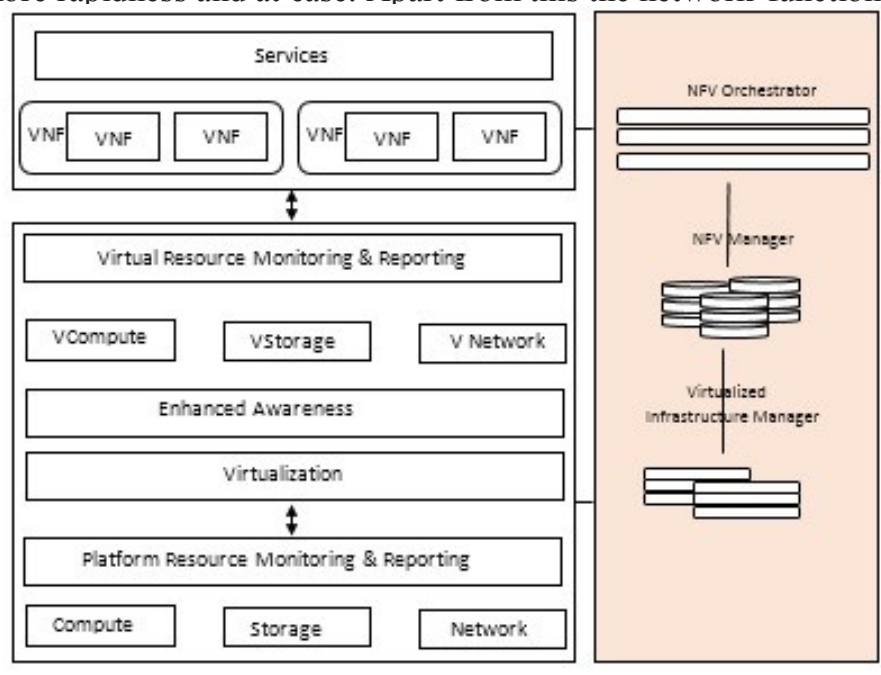

Fig 2 NFV 
Journal of ISMAC (2019)

Vol.01/ No. 03

Pages: 188-205

http://irojournals.com/iroismac/

DOI: https://doi.org/10.36548/jismac.2019.3.006

Provides an application level support for the devices, offering an enhanced quality of services, minimized power and space utilization.

\subsection{Deep Packet Inspection for SDN}

The Deep packet inspection shown in Fig 3 is to provide the packet inspection by continuously keeping track of the flow and the packets to determine the type of devices, duration of the service, the application types and the frequency used for establishing the connection so as to enhance the congestion control, minimized bandwidth utilization and the quality of service with payload inspection of the data packets. This provides the context awareness with selfadaptability for the devices in the network.

\begin{tabular}{|c|c|c|c|c|c|}
\hline \multicolumn{2}{|c|}{ Data Packet Header } & \multicolumn{4}{|c|}{ Data Packet Payload } \\
\hline $\begin{array}{l}\text { Origin and } \\
\text { target } \\
\text { address }\end{array}$ & $\begin{array}{l}\text { QOS, flow } \\
\text { Monitoring }\end{array}$ & Data & Audio Data & Video Data & $\begin{array}{l}\text { Threat and } \\
\text { interceptions }\end{array}$ \\
\hline
\end{tabular}

Fig 3 DPI

\subsection{SDN, NFV and DPI for virtual and sustainability in Wireless Network.}

The effective virtual wireless network with sustainability is obtained by converging the SDN, NFV and the DPI, as shown in the Fig 4 the deep packet inspection offered in the software based control layer of the SDN would enable an improved capabilities on analyzing, updating, scaling, according to the dynamic workloads. The Deep packet inspection in aid to the software defined networks enables to understand the large network services in the peer to peer applications, manages the network congestion, and guarantees the SLA (service level agreements) applications with enforcement and the policy definitions, and provides with the necessary security levels for the malware and the intrusions that are a threat. This deep packet inspection for the SDN allows the network to be application aware by helping in the identification, measurement and the optimization of the application. Further the Network function virtualization and the software defined networks allow a flexibility in the functioning of the network through the virtualization by adding numerous of devices and applications at ease and the flexibility in controlling with effective routing respectively. This accelerates the innovations in the service provisioning by the usage of standard virtualization techniques. So the converging of the SDN, NFV and DPI empowers the virtualization of the wireless network with 
Journal of ISMAC (2019)

Vol.01/ No. 03

Pages: 188-205

http://irojournals.com/iroismac/

DOI: https://doi.org/10.36548/jismac.2019.3.006

the greater reap overcoming the challenges in the resource allocation and embedding by extending support to the multiple service provider using the virtual networks that are heterogeneous, operating on multiple domains. The deep packet inspection that moves the network to be application aware thereby makes the network context aware with the self-adaptability according to the traffic flow.

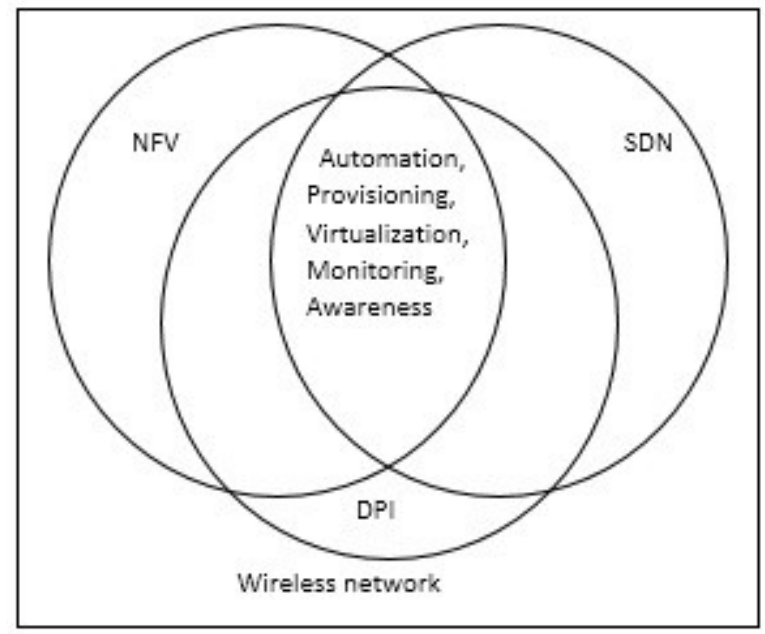

Fig 4 SDN, NFV and DPI for Wireless Network

\subsection{Sustainable virtual wireless network for Cloud and Enterprise Information System}

The merging of the SDN, NFV and DPI provides a virtual wireless network that is sustainable for the cloud and the enterprise information system by the service awareness provisioning, proper resource allocation overcoming the challenges by allowing the unified abstraction of the heterogeneous infrastructure such as storage, computation and the networking. Further the information's on the storage, computing and the network are bundled to provide with the global over view on abstraction and the control layer, initiates in the framing of the virtual networks by totally relying on the information bundle for the perfect service provisioning, meeting the requirements. The standard interfaces used enables the balanced precision and abstraction of the logical infrastructure view by limiting to the proper degree of state aggregation. The better analysis and the capabilities including the updating and scaling provisioning according to the changing workloads of the SDN in combination with the DPI enriches the virtual network with the centralized control and management of the networking, computing and storage provisioning across sovereign domains in the internet that are globally distributed. The integration moreover provides with the perfect distinction and the coordination in the embedding of the devices in the forwarding layer and their control and the management. In addition 
Journal of ISMAC (2019)

Vol.01/ No. 03

Pages: 188-205

http://irojournals.com/iroismac/

DOI: https://doi.org/10.36548/jismac.2019.3.006

assures with the isolation for the control among the multiple virtual networks embedded on the shared surface with elasticity on virtual network embedding with aid to scale-up, scale-down and co-migration.

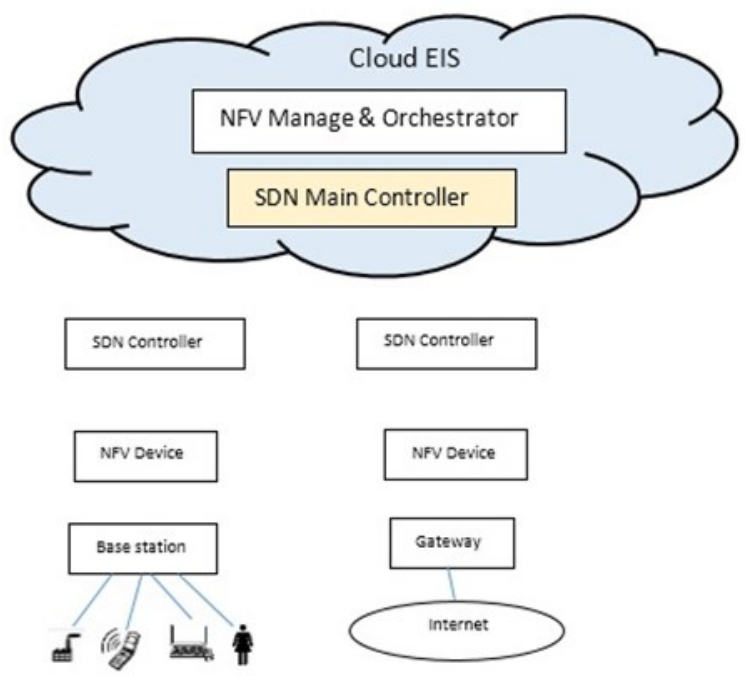

Fig 5 Sustainable Virtual Wireless Network for Cloud and EIS

The virtualization for sustainability in the cloud and the enterprise apart from service provisioning reducing the hardware used, cost and the down time, the virtual networks are energy aware and quality assured by the integrating of the SDN, NFV and DPI. The flexibility provisioning of the SDN and NFV and the packet header and the payload inspection of DPI empowers the proposed method of the virtual wireless network with the sustainability to achieve energy efficiency.

Thus the abstraction, the isolation provisioning and the packet inspection provisioning enabled by the software empowers the wireless virtual network in overcoming their challenges in the embedding over a physical structure and the resource allocation along with the numerous devices that are context aware and self-adapting to provide with the virtual network that are sustainable for the cloud and the enterprise information system thereby reducing the numerous hardware utilization by the replacing the dedicated hardware's, enhancing the storage facilities and shrinking the down time by the proper resource sharing across multiple servicing provisioning's with context awareness , self-adaptability and security provisioning using the SDN,NFV and DPI for the wireless virtual network to make them more sustainable. 
Journal of ISMAC (2019)

Vol.01/ No. 03

Pages: 188-205

http://irojournals.com/iroismac/

DOI: https://doi.org/10.36548/jismac.2019.3.006

The application of the SDN, NFV and DPI in conjunction further enables elasticity by the common hardware pooling and the shifting of workloads to meet the speedy bandwidth requirements on the multiple devices according to the user expectation.

\section{Result and Analysis}

The proposed virtual wireless network for sustainability using the SDN, NFV and DPI are simulated in the network simulator and validated to determine the efficiency of the same. The conjunction of the SDN and NFV causes the major reduction in the expenses, the different dedicated appliances, the slow deployment, the proper control, management, bandwidth utilization, congestion control and forwarding with routing facilitated by the open flow and the software implementation for the abstraction, isolation and removal causing easy migration and reduced operations. The DPI further helps in the monitoring of the header and the payload for the data packets to make the network context aware and protected from the unwanted malicious attacks. The comparison with the proposed model and the existing cloud models neglecting the integration of SDN, NFV and DPI is done to analyze the efficiency of the proposed Model and the quality of the service attained.

$\begin{array}{ll}\text { Parameter } & \text { Value } \\ \text { Simulation time } & 100 \text { Seconds } \\ \text { Allotted Area } & 1000 \mathrm{sq} \cdot \mathrm{m} \\ \text { Number of Services } & 500 \\ \text { Packet Size } & 1024 \\ \text { Packet Data Rate } & 1 \text { packet/second } \\ \text { Initial Energy } & 100 \text { joules }\end{array}$

Table. 1

\section{a) Network Throughput}

The Network throughput gives the essential bandwidth utilization by estimating the total completed services in the network, the flexible automation, service provisioning, virtualization, the context awareness, minimized bandwidth 
Journal of ISMAC (2019)

Vol.01/ No. 03

Pages: 188-205

http://irojournals.com/iroismac/

DOI: https://doi.org/10.36548/jismac.2019.3.006

utilization and the congestion control of the proposed model empowers it to achieve a sustained throughput without latency and the overhead which if incurred might cause congestion in the transmission.

The Fig 6 shows the throughput achievement of the proposed method. The further comparison with the existing model highlights the throughput enhancements acquired in the proposed.

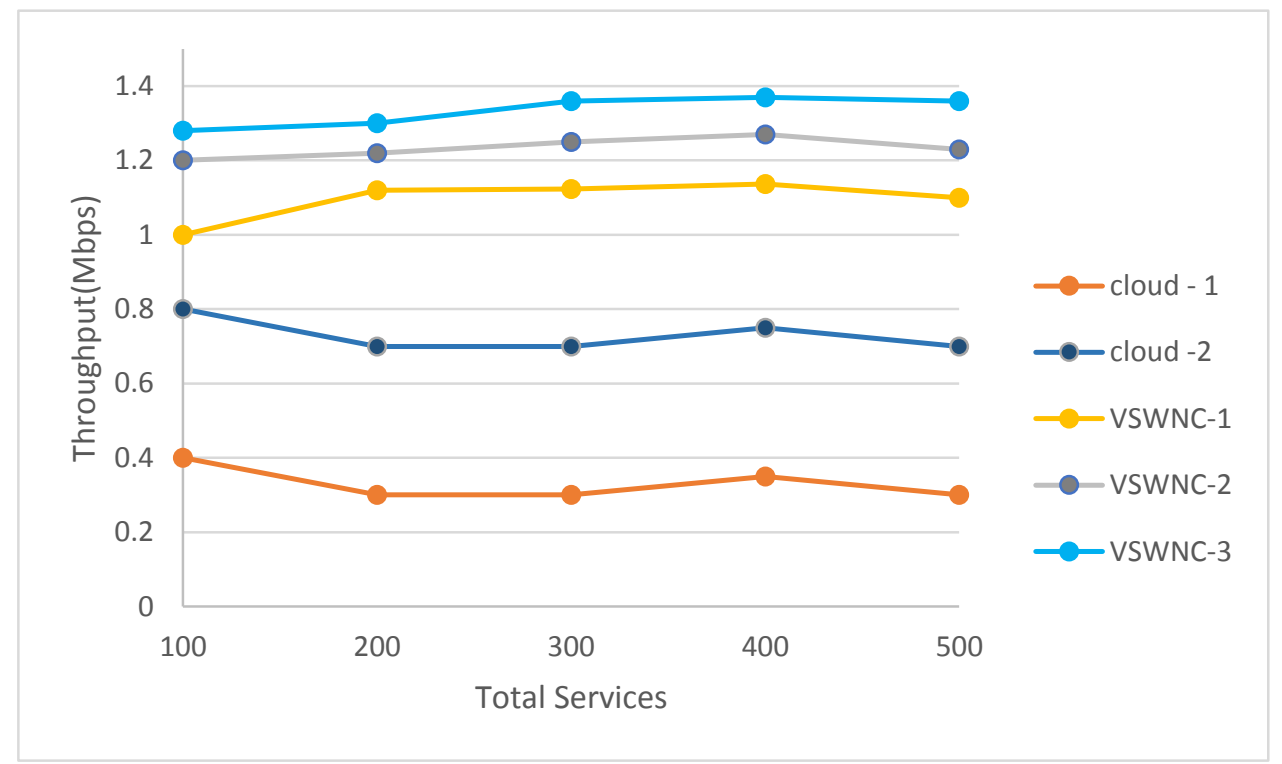

Fig 6 Network Throughput

\section{b) Network Delay}

Network delay being the essential performance characteristics in the network is used to determine the total travel time of the data from its origin to the end. The average measurement includes the time taken in the processing, transmission and the propagation. The virtualize network inclusion with SDN, NFV and DPI causes multiple transmissions, flexibly without being affected by latency and the malware intrusions. So the proposed model incurs less delay even on the increased number of services handling, due to the sustainable virtualization adapted in the network for the purpose of the service provisioning. 
Journal of ISMAC (2019)

Vol.01/ No. 03

Pages: 188-205

http://irojournals.com/iroismac/

DOI: https://doi.org/10.36548/jismac.2019.3.006

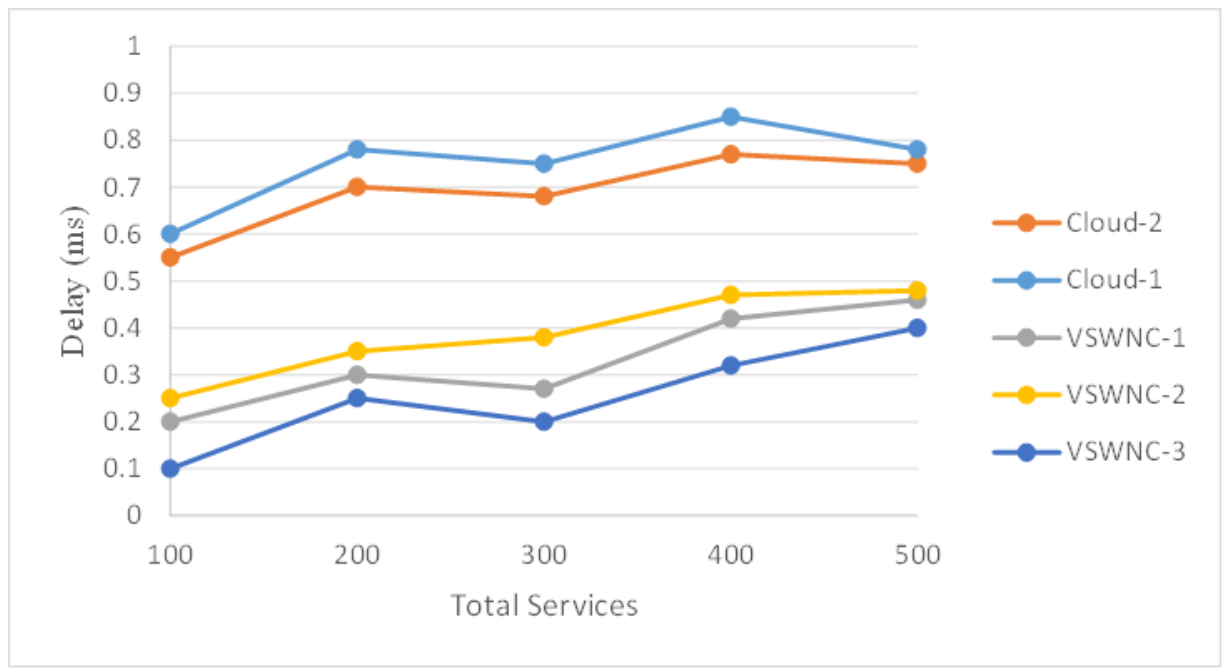

Fig 7 Network Delay

The Fig 7 gives the delay responses incurred in the proposed model for the varying number of services. Though the latency seems to be average at the beginning, as the number of service increases and depending on the type of the services there is a variation in the delay that is slightly high and then reduces to the normal level. This high delay incurred by the proposed is very much less compared to the very-high latency acquired in the existing model of cloud information system that neglects the SDN, NFV and DPI provisions.

\section{c) Network Packet Delivery Ratio}

The network packet delivery ratio is the performance metrics that provides the essential information on the successful transmission (services) that are completed, for the total number services on requisition. The proposed model with context awareness and self-adaptability allows the application and network aware, rapid service provisioning satisfying the user needs. More over the throughput and the delay enhancements attained, empowers the proposed model in acquiring a maximum network packet delivery ratio when compared to the other existing cloud models. 
Journal of ISMAC (2019)

Vol.01/ No. 03

Pages: 188-205

http://irojournals.com/iroismac/

DOI: https://doi.org/10.36548/jismac.2019.3.006

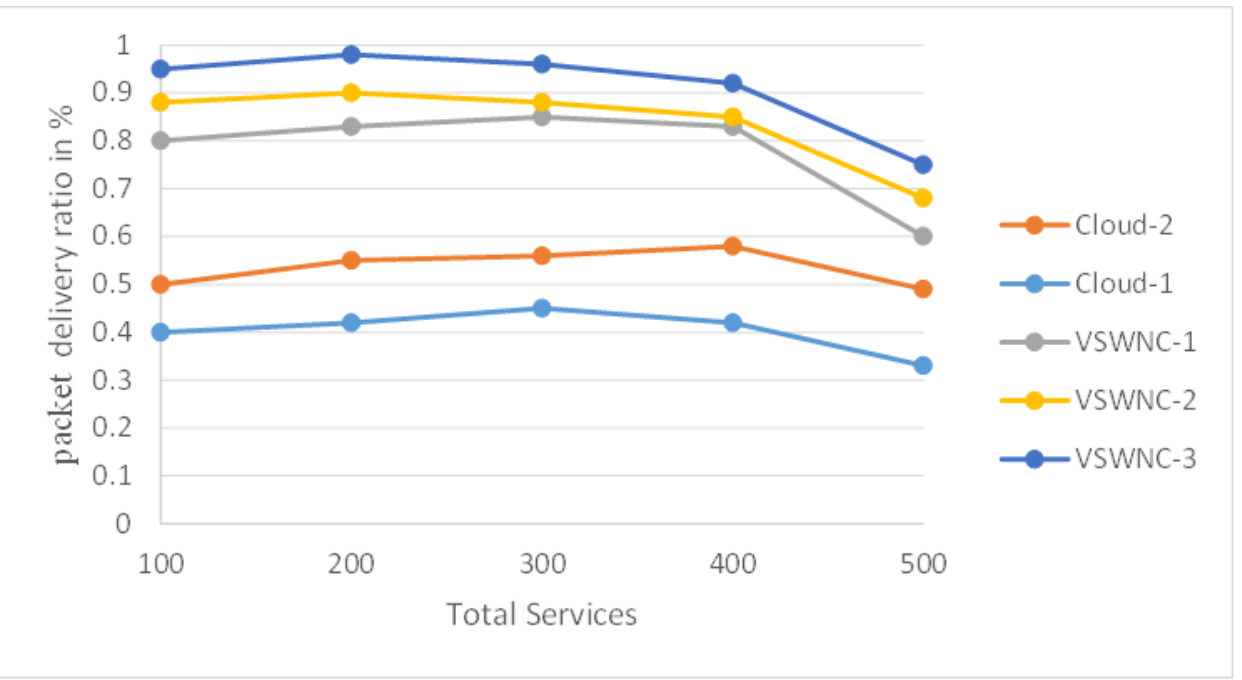

Fig 8 Network Packet Delivery Ratio

The Fig 8 details the PDR acquired on the proposed model for varying number and different types of services handled. The graph shows the maximum PDR achieved when compared to the existing models of cloud in the service provisioning. The successful service completion and the throughput enhancements go hand in hand in evincing the efficiency in the performance.

\section{d) Power Consumption}

The virtualization in the network that enables the usage of single resources to handle multiple works, causes much reduction in the hardware utilization. The reduced hardware resources results with the reduction in the power consumption, which would be very high on network neglecting the virtualization. Further the integrated process of automation, service provisioning, context awareness and the flow monitoring does a considerable reduction in the utilization of the power which would otherwise be wasted on the separate service identification and securing of the data. 
Journal of ISMAC (2019)

Vol.01/ No. 03

Pages: 188-205

http://irojournals.com/iroismac/

DOI: https://doi.org/10.36548/jismac.2019.3.006

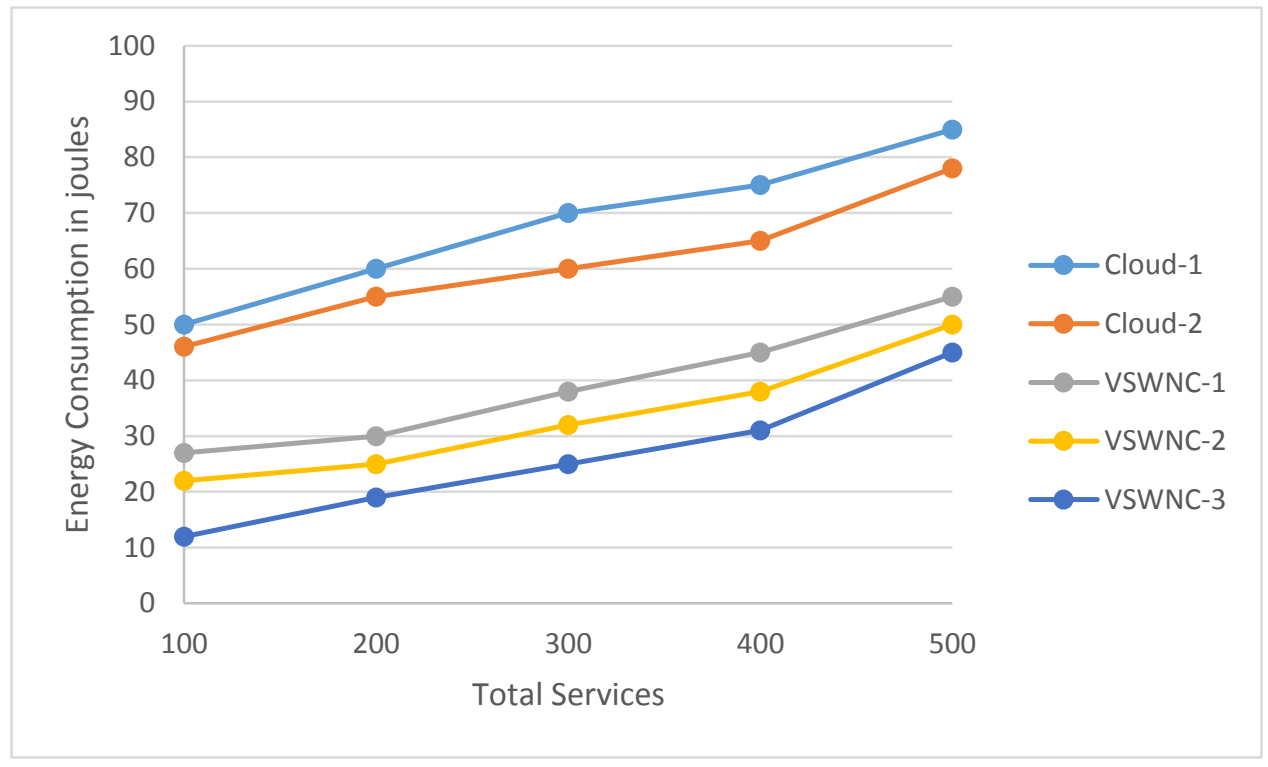

Fig 9 Power Consumption

The Fig 9 shows the power consumption graph of the virtualized network including the SDN, NFV and DPI shows improvement when compared to the existing methods neglecting the inclusion of the automation, virtualization, flow monitoring and a secured service provisioning.

\section{e) Packet Loss Ratio}

This provides the measure of losses incurred during the provisioning of the services. The above simulation results achieved on the throughput, delay and packet delivery ratio shows that the losses incurred in the proposed model is very much less when compared to the existing methodologies. The further simulation for measuring the packet losses is shown in the Fig 10. 
Journal of ISMAC (2019)

Vol.01/ No. 03

Pages: 188-205

http://irojournals.com/iroismac/

DOI: https://doi.org/10.36548/jismac.2019.3.006

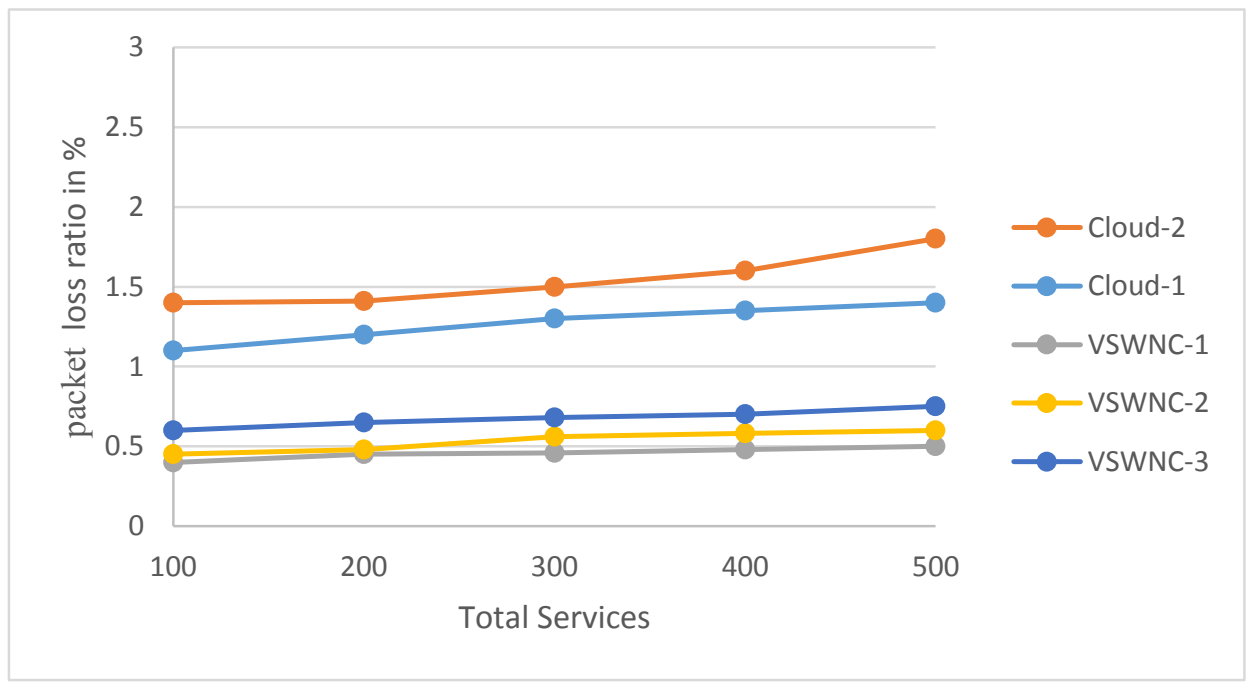

Fig 10 Packet Loss Ratio

The Fig 10 gives the packet loss incurred by the virtualized network that is much less compared to the preceding methods that incur a loss of more than $1 \%$. So the proposed model enriches the network with less losses in the service handling and proves to be efficacious.

\section{f) Cost Optimization}

The actual purpose on virtualization is for having an optimization on the total expense. The proposed system offering the integrated automation, virtualization in the resources and flow monitoring services, with software abstraction and isolation further cut downs the enormous cost that would be incurred by the system that handles all the above mentioned tasks separately. 
Journal of ISMAC (2019)

Vol.01/ No. 03

Pages: 188-205

http://irojournals.com/iroismac/

DOI: https://doi.org/10.36548/jismac.2019.3.006

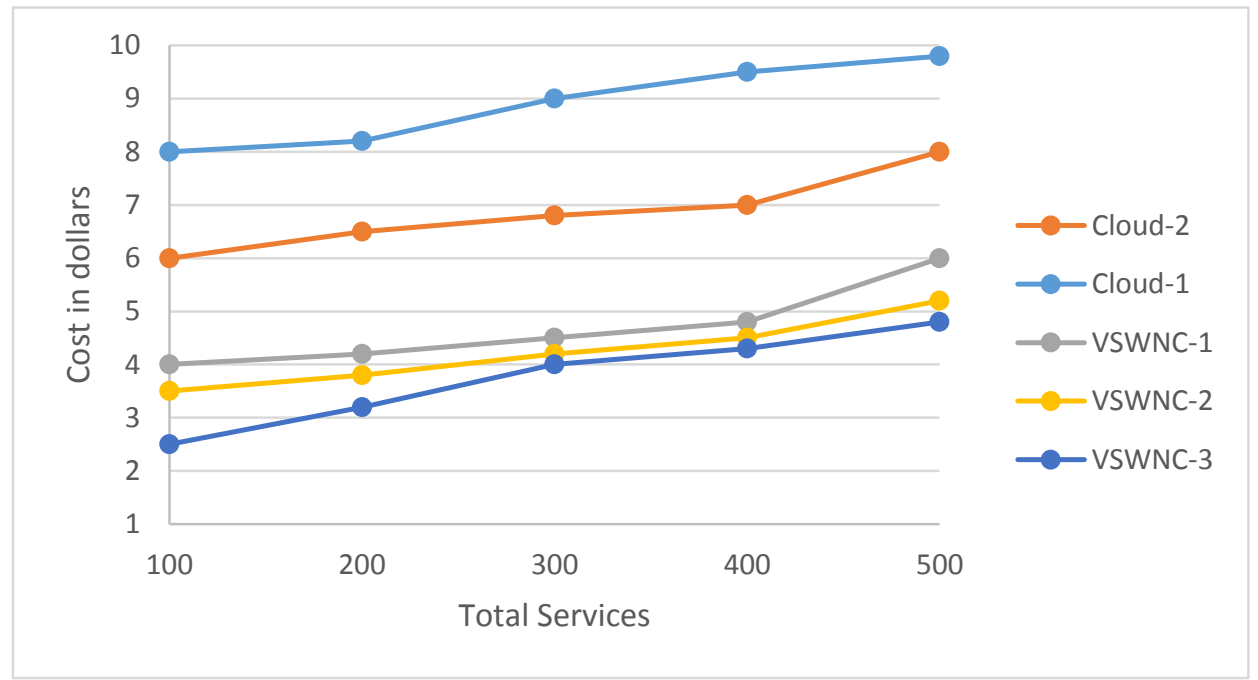

Fig 11 Cost Optimization

The graph obtained in the Fig 11 gives the cost incurred per second on the service provisioning of the network. The proposed method shows an enhanced cost optimization when compared to the preceding methods. The simulation results acquired proves the sustainability of the network and its efficient service handling on employing with the cloud services and the enterprise information system satisfying the customer needs according to their desired requirements.

\section{Conclusion}

The Proposed method of SDN,NFV,DPI integration with the wireless network proffering a flexible automation with, virtualization, and flow monitoring respectively, offers an easy resource sharing, migration and security provisions in rendering services along with the cloud and the enterprise information system, further the application and the network awareness causes easy identification of the service types and easy service provisioning with the minimized number of hardware devices enabled by the resource sharing. More over the software abstraction and the isolation helps in the easy migration to the newly developed technologies. The flow monitoring enables the secured servicing provisioning made possible in the proposed method. Thus the virtual wireless network is sustained in its performance, with the inclusion of the above features of SDN, NFV and DPI. The cloud and the enterprise information system with the sustainable virtual wireless network evinced to be very efficacious in terms of quality of service, successful transmission and cost effectiveness on validating the proposed model in the network Simulator. 
Journal of ISMAC (2019)

Vol.01/ No. 03

Pages: 188-205

http://irojournals.com/iroismac/

DOI: https://doi.org/10.36548/jismac.2019.3.006

\section{References}

[1] Rajesh, V., J. M. Gnanasekar, R. S. Ponmagal, and P. Anbalagan. "Integration of wireless sensor network with cloud." In 2010 International Conference on Recent Trends in Information, Telecommunication and Computing, pp. 321-323. IEEE, 2010.

[2] Irish, Althiya Eby, Sebastian Terence, and Jude Immaculate. "Efficient Data Collection Using Dynamic Mobile Sink in Wireless Sensor Network." In Wireless Communication Networks and Internet of Things, pp. 141-149. Springer, Singapore, 2019.

[3] Khan, Imran, Fatna Belqasmi, Roch Glitho, Noel Crespi, Monique Morrow, and Paul Polakos. "Wireless sensor network virtualization: A survey." IEEE Communications Surveys \& Tutorials 18, no. 1 (2016): 553576.

[4] Fontes, Ramon R., Samira Afzal, Samuel HB Brito, Mateus AS Santos, and Christian Esteve Rothenberg. "Mininet-WiFi: Emulating software-defined wireless networks." In 2015 11th International Conference on Network and Service Management (CNSM), pp. 384-389. IEEE, 2015.

[5] Khan, Imran, Rifat Jafrin, Fatima Zahra Errounda, Roch Glitho, Noël Crespi, Monique Morrow, and Paul Polakos. "A data annotation architecture for semantic applications in virtualized wireless sensor networks." In 2015 IFIP/IEEE International Symposium on Integrated Network Management (IM), pp. 27-35. IEEE, 2015.

[6] Nkomo, Malvin, Gerhard P. Hancke, Adnan M. Abu-Mahfouz, Saurabh Sinha, and Adeiza J. Onumanyi. "Overlay virtualized wireless sensor networks for application in industrial internet of things." (2018).

[7] Khan, Imran, Jagruti Sahoo, Son Han, Roch Glitho, and Noël Crespi. "A genetic algorithm-based solution for efficient in-network sensor data annotation in virtualized wireless sensor networks." In 2016 13th IEEE Annual Consumer Communications \& Networking Conference (CCNC), pp. 321-322. IEEE, 2016.

[8] Arjun, D. S., Arunachalam Bala, V. Dwarakanath, K. S. Sampada, Prahlada Rao BB, and Haribabu Pasupuleti. "Integrating cloud-WSN to analyze weather data and notify SaaS user alerts during weather disasters." In 2015 IEEE International Advance Computing Conference (IACC), pp. 899-904. IEEE, 2015.

[9] Raee, Vahid Maleki, Diala Naboulsi, and Roch Glitho. "Energy Efficient Task Assignment in Virtualized Wireless Sensor Networks." In 2018 IEEE Symposium on Computers and Communications (ISCC), pp. 00976-00979. IEEE, 2018.

[10] Kaiwartya, Omprakash, Abdul Hanan Abdullah, Yue Cao, Jaime Lloret, Sushil Kumar, Rajiv Ratn Shah, Mukesh Prasad, and Shiv Prakash. "Virtualization in wireless sensor networks: Fault tolerant embedding for internet of things." IEEE Internet of Things Journal 5, no. 2 (2018): 571-580.

[11]Han, Bo, Vijay Gopalakrishnan, Lusheng Ji, and Seungjoon Lee. "Network function virtualization: Challenges and opportunities for innovations." IEEE Communications Magazine 53, no. 2 (2015): 90-97. 
Journal of ISMAC (2019)

Vol.01/ No. 03

Pages: 188-205

http://irojournals.com/iroismac/

DOI: https://doi.org/10.36548/jismac.2019.3.006

[12] Kumar, M. Sandeep. "Research and development of virtualization in wireless sensor networks." JOIV: International Journal on Informatics Visualization 2, no. 2 (2018): 96-103.

[13] Niebert, Norbert, Ibtissam El Khayat, Stephan Baucke, Ralf Keller, René Rembarz, and Joachim Sachs. "Network virtualization: A viable path towards the future internet." Wireless Personal Communications 45, no. 4 (2008): 511-520.

[14] Liang, Chengchao, F. Richard Yu, and Xi Zhang. "Information-centric network function virtualization over 5G mobile wireless networks." IEEE network 29, no. 3 (2015): 68-74.

[15]Feng, Zhiyong, Chen Qiu, Zebing Feng, Zhiqing Wei, Wei Li, and Ping Zhang. "An effective approach to 5G: Wireless network virtualization." IEEE Communications Magazine 53, no. 12 (2015): 53-59.

[16] Romero, David, and François Vernadat. "Enterprise information systems state of the art: Past, present and future trends." Computers in Industry 79 (2016): 3-13.

[17] Petrasch, Roland, and Roman Hentschke. "Cloud storage hub: Data management for IoT and industry 4.0 applications: Towards a consistent enterprise information management system." In 2016 Management and Innovation Technology International Conference (MITicon), pp. MIT-108. IEEE, 2016.

[18]Fang, Shifeng, Li Da Xu, Yunqiang Zhu, Jiaerheng Ahati, Huan Pei, Jianwu Yan, and Zhihui Liu. "An integrated system for regional environmental monitoring and management based on internet of things." IEEE Transactions on Industrial Informatics 10, no. 2 (2014): 1596-1605.

[19]Zhang, Da-Wei, Fu-Quan Sun, Xu Cheng, and Chao Liu. "Research on hadoop-based enterprise file cloud storage system." In 2011 3rd International Conference on Awareness Science and Technology (iCAST), pp. 434-437. IEEE, 2011.

[20] Jun, Chen, and Ma Yan Wei. "The research of supply chain information collaboration based on cloud computing." Procedia Environmental Sciences 10 (2011): 875-880. 